Miriam Mattoscio, MD

Richard Nicholas, FRCP,

$\mathrm{PhD}$

Maria P. Sormani

Omar Malik, FRCP, PhD

Jean S. Lee, MRCP, MA

Adam D. Waldman, MD,

$\mathrm{PhD}$

Francesco Dazzi, MD

Paolo A. Muraro, MD,

$\mathrm{PhD}$

Correspondence to

Dr. Muraro:

p.muraro@imperial.ac.uk

Supplemental data at Neurology.org

\section{Hematopoietic mobilization}

\section{Potential biomarker of response to natalizumab in multiple sclerosis}

\section{ABSTRACT}

Objective: To ascertain the mobilization from the bone marrow and the functional relevance of the increased number of circulating hematopoietic stem and progenitor cells (HSPC) induced by the anti- $\alpha-4$ integrin antibody natalizumab in patients with multiple sclerosis (MS).

Methods: We evaluated CD45 ${ }^{\text {low }}$ CD34+ HSPC frequency by flow cytometry in blood from 45 natalizumab-treated patients (12 of whom were prospectively followed during the first year of treatment as part of a pilot cohort and 16 prospectively followed for validation), 10 untreated patients with MS, and 24 healthy donors. In the natalizumab-treated group, we also assessed sorted HSPC cell cycle status, T- and B-lymphocyte subpopulation frequencies ( $\mathrm{n}=29)$, and HSPC differentiation potential $(n=10)$.

Results: Natalizumab-induced circulating HSPC were predominantly quiescent, suggesting recent mobilization from the bone marrow, and were capable of differentiating ex vivo. Circulating HSPC numbers were significantly increased during natalizumab, but heterogeneously, allowing the stratification of mobilizer and nonmobilizer subgroups. Nonmobilizer status was associated with persistence of disease activity during treatment. The frequency of B cells and CD103+CD8+ regulatory $T$ cells persistently increased, more significantly in mobilizer patients, who also showed a specific naive/memory B-cell profile.

Conclusions: The data suggest that natalizumab-induced circulating HSPC increase is the result of true mobilization from the bone marrow and has clinical and immunologic relevance. HSPC mobilization, associated with clinical remission and increased proportion of circulating $B$ and regulatory T cells, may contribute to the treatment's mode of action; thus, HSPC blood counts could represent an early biomarker of responsiveness to natalizumab. Neurology ${ }^{\circledR}$ 2015;84:1473-1482

\section{GLOSSARY}

AFFIRM = Natalizumab Safety and Efficacy in Relapsing-Remitting Multiple Sclerosis; DMT = disease-modifying therapies; EDSS = Expanded Disability Status Scale; $\mathbf{H D}=$ healthy donors; HSPC = hematopoietic stem and progenitor cells; IgD = immunoglobulin D; $\mathbf{M S}=$ multiple sclerosis; PB = peripheral blood; PBMC = peripheral blood mononuclear cell; $\mathbf{P M L}=$ progressive multifocal encephalopathy; SENTINEL = Safety and Efficacy of Natalizumab in Combination With Interferon Beta-1a in Patients With Relapsing-Remitting Multiple Sclerosis.

Multiple sclerosis (MS) is thought to be initiated by inflammatory blood-derived leukocytes entering the CNS. $\alpha 4 \beta 1$ integrin plays a key role in leukocyte trafficking ${ }^{1}$; its $\alpha 4$ subunit is targeted by the MS treatment natalizumab, which suppresses brain MRI lesion formation and reduces relapse rate. ${ }^{2-4}$ Improvement of neurologic function in treated patients has also been observed..$^{5-7}$ Natalizumab's anti-inflammatory effect in CNS is mirrored by a radical decrease of lymphocyte number in the $\mathrm{CSF}^{8,9}$ and by T-cell sequestration in the periphery, ${ }^{10,11}$ yet less is known about its effects on B cells and on hematopoietic stem and progenitor cells (HSPC) recirculation, both cell types expressing $\alpha 4 \beta 1$. Natalizumab treatment induces a significant and protracted peripheral HSPC increase in the blood of patients with MS. ${ }^{12,13}$ The mechanism and the functional significance of the observed increase, however, remain poorly understood.

From the Department of Medicine, Division of Brain Sciences, Centre for Neuroscience, Wolfson Neuroscience Laboratories (M.M., R.N., O.M., P.A.M.), and the Department of Medicine, Division of Experimental Medicine, Centre for Haematology (F.D.), Imperial College London, UK; the Departments of Neurosciences (R.N., O.M.) and Imaging (J.S.L., A.D.W.), Imperial College Healthcare NHS Trust, London, UK; and the Biostatistics Unit, Department of Health Sciences (M.P.S.), University of Genoa, Italy.

Go to Neurology.org for full disclosures. Funding information and disclosures deemed relevant by the authors, if any, are provided at the end of the article. 
The objective of this study was to ascertain whether the HSPC increase induced by natalizumab treatment is consequent to mobilization from the bone marrow and whether the increase has a relationship with the clinical response to treatment and immune homeostasis in patients with MS. Therefore we evaluated circulating HSPC cell cycle status, frequency, and differentiation potential, and measured $\mathrm{T}$ - and B-cell subsets proportion in natalizumab-treated patients with MS, untreated patients with MS, and healthy donors (HD). We then tested for potential correlations of HSPC and immune variables with clinical and MRI parameters of response to natalizumab.

METHODS Standard protocol approvals, registrations, and patient consents. This research study had ethical approval (REC Ref. 09/H0707/18) and written informed consent was obtained from all participants.

Subjects, blood sampling, and clinical assessments. We recruited 45 subjects with MS who received treatment with natalizumab, given IV every 4 weeks, at Charing Cross Hospital, London, UK. Ten untreated patients with MS and $24 \mathrm{HD}$ served as controls. Peripheral blood $(\mathrm{PB})$ was taken between 8:30 AM and 12:30 PM from all subjects, thus avoiding potential circadian time-related variation. ${ }^{14}$ All natalizumab-treated patients were monitored every 6 months for clinical response to therapy with assessment of Expanded Disability Status Scale (EDSS) score and MRI.

The demographic and clinical characteristics of all study groups and the schedule of PB sampling are given in table e-1 on the Neurology ${ }^{\circledR}$ Web site at Neurology.org.

Cell staining for flow cytometry. Cell surface immunostaining for HSPC enumeration and for T- and B-lymphocyte phenotyping by flow cytometry was performed longitudinally on freshly collected whole $\mathrm{PB}$ and freshly isolated PB mononuclear cell (PBMC) samples, respectively.

Stained samples were acquired on a 2-laser, 4-color FACS Calibur flow-cytometer (Becton Dickinson, Franklin Lakes, NJ). Data were analyzed using Cell Quest (Becton Dickinson) and FlowJo software (TreeStar, Ashland, OR)

Other methods. Further methodologic details and all statistical methods are provided in appendix e- 1 .

RESULTS Natalizumab-induced circulating HSPC are predominantly quiescent and retain full expansion capacity ex vivo. The first aim of our study was to address whether HSPC are mobilized from the bone marrow and retain intact differentiating capacity during natalizumab treatment. To assess whether circulating HSPC from patients receiving natalizumab share the physiologic quiescent proliferation status of bone marrow-resident HSPC, we examined the cell cycle status of HSPC magnetically sorted (average purity 90\%) from PBMC of the first 29 natalizumabtreated patients with MS consecutively recruited in the study (12 prospective and 17 cross-sectional), of 10 untreated patients with MS, and of $24 \mathrm{HD}$. One typical example of a flow cytometry acquisition plot is shown in figure 1A. Cell cycle analysis showed a significantly higher proportion of quiescent HSPC (G0 status) in the natalizumab-treated crosssectional group compared to the untreated group $(p=0.0003$; figure 1B). Also, the proportion of quiescent cells was significantly more homogeneous in the natalizumab-treated patients than in $\mathrm{HD}$ ( $F$ test for variance $p<0.001$ ).

We then tested Lin-CD34+-sorted HSPC of 10 natalizumab-treated patients (method described in appendix e-1) for ex vivo differentiation capacity with a colony-forming units assay and observed functional expansion of natalizumab-induced HSPC (images in figure 1C) comparable to those from HD ( $n=3)$.

These results demonstrated that the natalizumabinduced circulating HSPC are predominantly and homogeneously quiescent, thus more similar to HSPC in the bone marrow than the circulating HSPC in untreated patients, supporting the notion that natalizumab treatment actively increases the egress of HSPC from the bone marrow, rather than simply blocking their extravasation. Also, natalizumab-induced HSPC of patients with MS are "healthy" and expand at the same rate as HSPC of HD.

CD34+ HSPC mobilization responses vary among natalizumab-treated patients with MS. To ascertain and quantify the increase of circulating HSPC following natalizumab treatment, we enumerated them in treated and untreated patients with MS and in HD. CD34+ HSPC absolute counts were significantly higher in the blood of natalizumab-treated, crosssectionally evaluated patients with MS ( $\mathrm{n}=17$; after 5 mean infusions, range $1-30$ ), compared to untreated patients with MS ( $\mathrm{n}=10 ; p<0.05$; figure $2 \mathrm{~A}$ ). The HD ( $\mathrm{n}=24)$ had intermediate CD34+ HSPC counts. We also detected a significantly greater variance of the CD34+ HSPC counts in the treated cross-sectional cohort compared to the untreated group ( $F$ test $p=0.0009)$ and to the HD $(p<0.0001)$, unrelated to the number of infusions received.

We next measured circulating CD34+ HSPC numbers longitudinally over 1 year in patients recruited prospectively before starting natalizumab. Among the first 12 consecutive patients and constituting the original pilot cohort, we observed a bimodal response, with $6 / 12$ patients showing a significantly higher HSPC count of $\geq$ twofold compared to baseline values after 2 infusions. When we looked at a second group of 16 patients newly recruited into the study to serve as a validation cohort, we also observed a variable response, with $12 / 16 \mathrm{pa}-$ tients showing $\geq$ twofold increase of the HSPC 
Figure 1 Natalizumab-induced circulating hematopoietic stem and progenitor cells are predominantly quiescent and retain full expansion capacity ex vivo

A

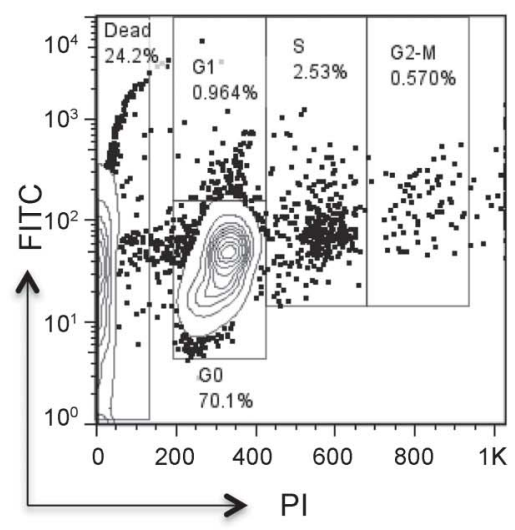

$\mathrm{B}$

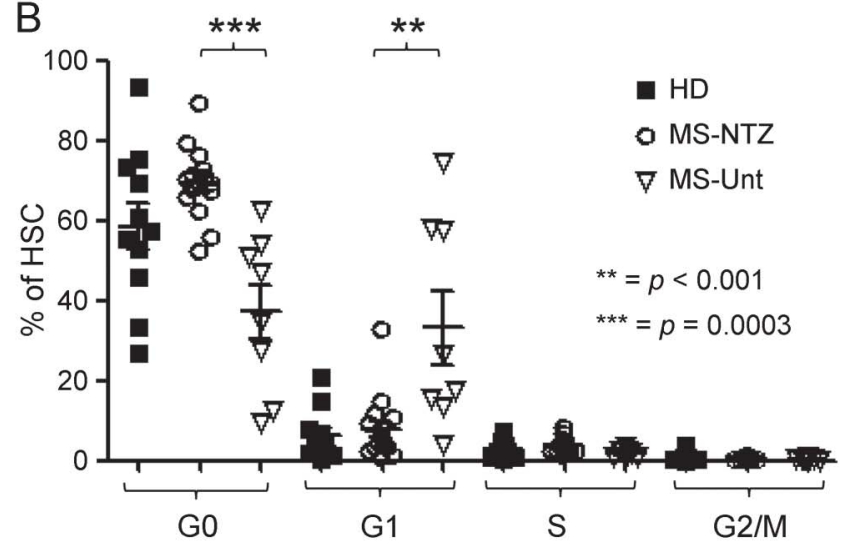

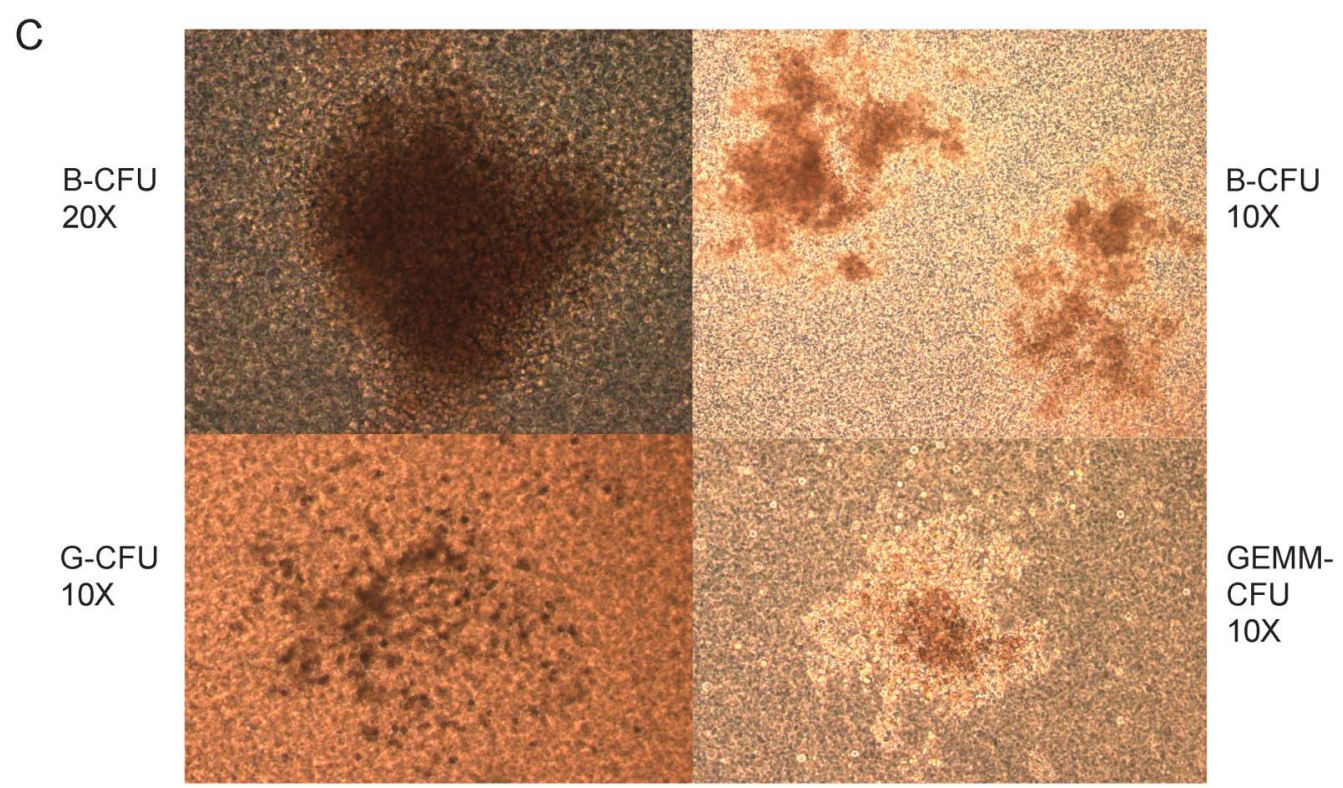

(A) Representative example of flow cytometry acquisition plot shows CD34+ hematopoietic stem and progenitor cells (HSPC) magnetically sorted from peripheral blood mononuclear cells of 1 patient who had received 2 infusions of natalizumab, after labeling with fluorescein isothiocyanate and propidium iodide to measure intracellular protein and DNA content, respectively; GO, G1, S, and G2/M phases of the cell cycle are defined according to the expression of the 2 markers. (B) Cell cycle analysis shows a significantly higher proportion of quiescent (GO) HSPC in natalizumab-treated patients with multiple sclerosis (MS-NTZ) than in untreated patients (MS-Unt; mean \pm SD: MS-NTZ $69.33 \pm 8.89 \%$; MS-Unt $37.09 \pm 19.58 \%$ ). The fraction of HSPC in the first phase within the interphase (G1) prevailed in the untreated compared to the natalizumabtreated patients. Also, circulating HSPC appear to be more homogenously quiescent in the natalizumab-treated patients than in the healthy donors (HD) (proportion of HSPC in GO: mean \pm SD $58.56 \pm 19.2 \%$ ), the latter group showing significantly higher variance. (C) Representative inverted light microscopy images of colony-forming units (CFU) derived from natalizumab-induced HSPC; blast (B), granulocyte (G), and granulocyte erythrocyte monocyte megakaryocyte (GEMM) CFU types are shown.

count. To increase the statistical power, we pooled together the two (pilot and validation) cohorts of prospectively followed patients and analyzed the results in the total population $(n=28)$. Circulating CD34+ HSPC counts significantly increased 1 month after the first natalizumab infusion compared to prenatalizumab baseline $(p<0.005)$ and the increase persisted after $2(p<0.005), 6(p<0.0001)$, and $12(p<$ $0.05)$ infusions (figure $2 \mathrm{~A}$ ).
Significantly higher circulating HSPC counts compared to the untreated patients group were first detected in prospective natalizumab-treated patients after 2 infusions $(p<0.05)$. Thus, the 2 months on treatment time point was identified as the earliest to reliably separate HSPC mobilization response. We therefore calculated the fold change of HSPC absolute count after 2 infusions compared to baseline and utilized the geometric mean (2.1) to define the 
Figure 2 CD34+ hematopoietic stem and progenitor cells mobilization responses vary among natalizumabtreated patients with multiple sclerosis

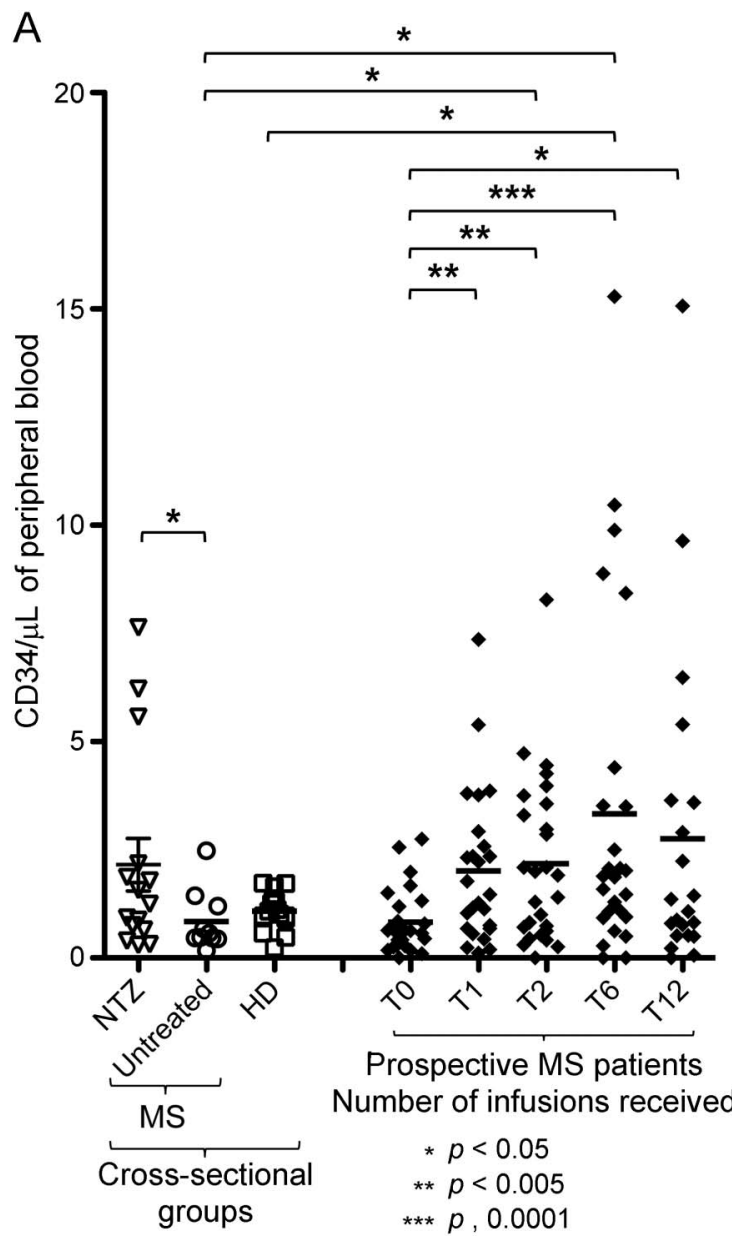

B

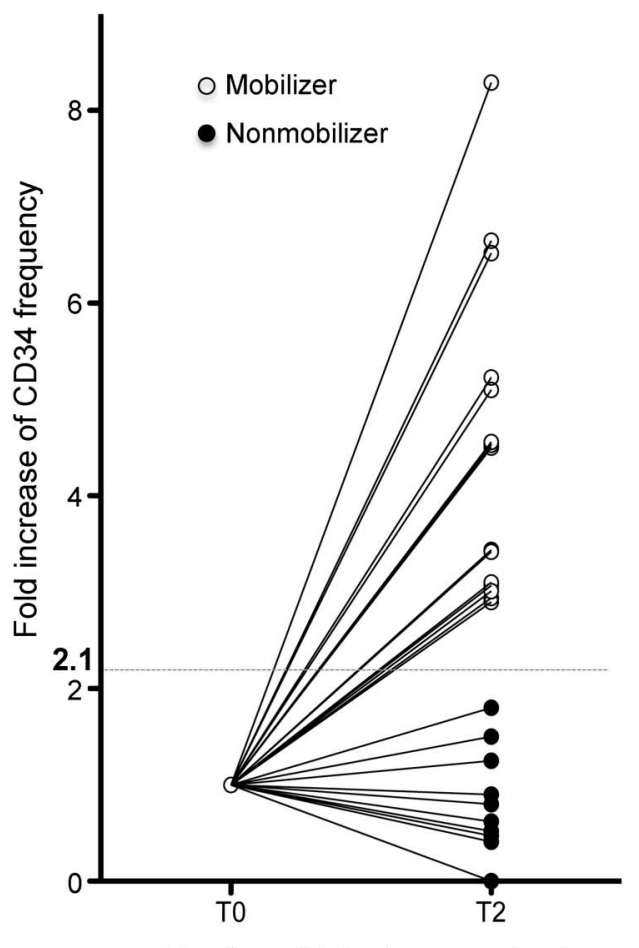

Number of infusions received

(A) In a cross-sectional group comparison (left side), CD34+ hematopoietic stem and progenitor cells (HSPC) counts are significantly increased in the natalizumab-treated population (multiple sclerosis [MS]-NTZ; mean \pm SD $2.16 \pm 2.21$ CD34+

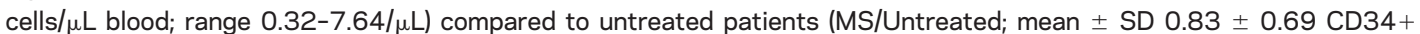
cells $/ \mu \mathrm{L}$ blood; range $0.17-2.48 / \mu \mathrm{L})$. The HSPC counts for the healthy donors (HD) control group are also shown for reference (mean \pm SD $0.95 \pm 0.43 \mathrm{CD} 34+$ cells/ $\mu \mathrm{L}$ blood; range $0.24-1.73 / \mu \mathrm{L}$ ). In a prospective evaluation, significant increases of circulating CD34+ HSPC absolute number are seen in the natalizumab-treated group at all time points during treatment (after 1, 2, 6, and 12 infusions) compared to baseline. CD34+ HSPC mean count \pm SD: at baseline, $0.81 \pm 0.7$

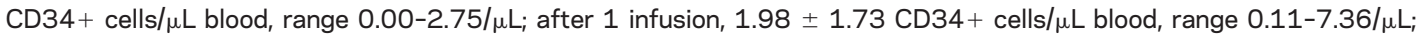
after 2 infusions, $2.11 \pm 1.91 \mathrm{CD} 34+$ cells $/ \mu \mathrm{L}$ blood, range $0.00-8.28 / \mu \mathrm{L}$; after 6 infusions, $3.12 \pm 3.77 \mathrm{CD} 34+\mathrm{cells} / \mu \mathrm{L}$

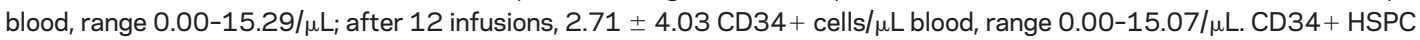
counts appear to be widely dispersed in distribution among all natalizumab-treated patient groups, regardless of the number of infusions received. (B) Individual CD34+ HSPC counts fold increase from baseline to 2 natalizumab infusions is shown for the prospectively followed patients group $(n=27)$ and reveals clear interindividual differences. The geometrical mean fold change was employed as unbiased cutoff value (dotted line $=2.1$ ) to stratify mobilizer patients (fold change of circulating HSPC count $\geq 2.1 ; n=17$; open circles) and nonmobilizer patients (fold change $<2.1 ; n=10$; filled circles).

fold change cutoff value to stratify the individuals. Patients who showed $a \geq 2.1$-fold change in their HSPC absolute count after 2 natalizumab infusions compared to baseline were defined as mobilizers, whereas those who showed a $<2.1$-fold change were defined as nonmobilizers (figure $2 \mathrm{~B}$ ).

After excluding one patient whose baseline HSPC count was null (thus preventing the quantification of fold change), among the remaining 27 patients examined 10 were non-mobilizers and 17 were mobilizers (table e-2). The patient stratification was utilized for the clinical and immunologic correlation analyses.

HSPC nonmobilizer patients show persistent MRI and clinical disease activity during natalizumab treatment. To assess whether HSPC mobilizer and nonmobilizer patient status was associated with different responses to natalizumab, we examined clinical and MRI measures in the 2 patient strata. Among the 27 prospective patients with the required MRI data, the number of 
brain gadolinium-enhancing T1 lesions at 6 months MRI during treatment was significantly higher in the nonmobilizer patients $(\mathrm{n}=10$ patients; mean \pm SD $5 \pm 6.53$ lesions, range 0-20) than in the mobilizer patients $(\mathrm{n}=17$ patients; mean \pm SD $1 \pm 1.34$ lesions, range $0-3 ; p=0.002$ ). The difference remained borderline significant $(p=0.059)$ after adjusting for the baseline values, when nonmobilizer patients had higher, albeit nonsignificantly, number of gadolinium-enhancing T1 lesions (mean \pm SD 3.89 \pm 5.60, range $0-15$ ) than mobilizers (mean \pm SD $0.94 \pm 1.44$, range $0-4 ; p=0.41$ ). The contrasting change in the number of T1 gadolinium-enhancing lesions between baseline and 6 months MRI in the mobilizer (decrease) and nonmobilizer patients (increase) is shown in figure 3A. Accordingly, the proportion of patients showing persistence of one or

Figure 3 Persistent multiple sclerosis disease activity during natalizumab treatment in nonmobilizer patients
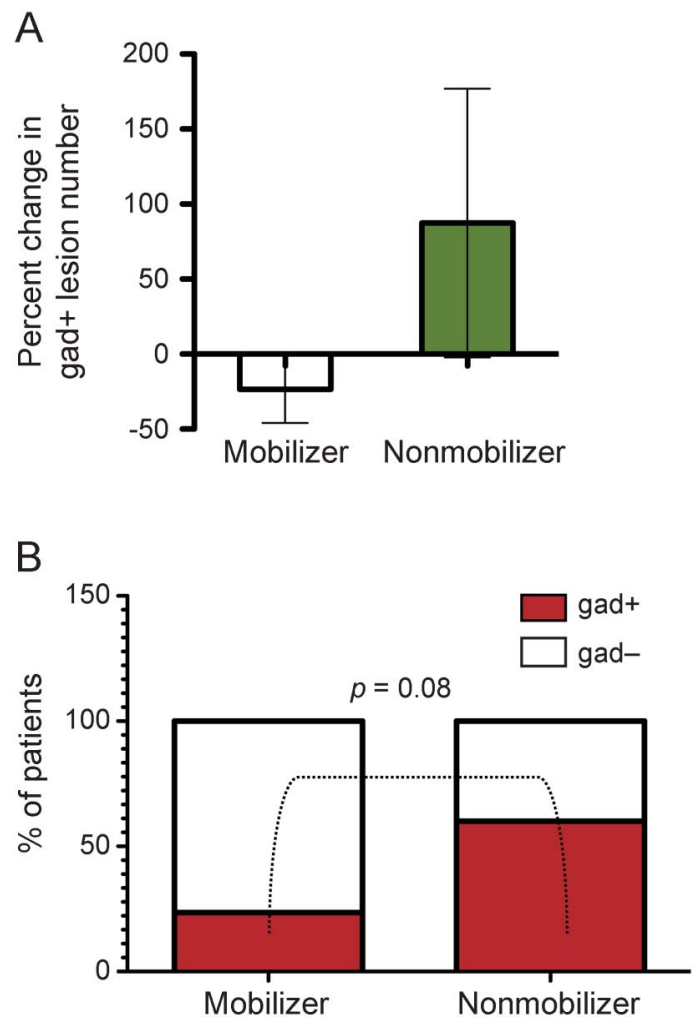

(A) An opposite change in the number of brain gadoliniumenhancing $(\mathrm{gad}+)$ T1 lesions between pretreatment baseline and after 6 natalizumab infusions was seen in the patient subgroups, with a decrease in mobilizer $(n=17)$ and an increase in nonmobilizer patients $(n=10)$. The histogram bars represent the percent variation and the whiskers indicate the 95\% confidence intervals. (B) The proportion of patients who had brain gad $+\mathrm{T} 1$ lesions after 6 natalizumab infusions was higher in the nonmobilizer ( $\mathrm{n}=6 / 10,60 \%)$ than in the mobilizer group $(\mathrm{n}=4 / 17$, $23.5 \%)$ but the difference did not reach statistical significance $(p=0.08)$. more T1 gadolinium-enhancing lesions at 6 months on treatment was higher in the nonmobilizer cohort $(\mathrm{n}=6 / 10,60 \%)$ than in the mobilizer one $(\mathrm{n}=4 / 17$, $23.5 \%$; figure $3 \mathrm{~B}$ ) although, probably due to the small number of subjects, the difference was not statistically significant $(p=0.08)$. In line with the postgadolinium MRI findings, a nonsignificant trend towards higher new T2 lesion numbers at 6 months of treatment (available for 23 of the 27 patients, stratified in 10 nonmobilizers and 13 mobilizers) was found in nonmobilizer patients (mean \pm SD $2.8 \pm 5.5$, range $0-16$ ) compared to the mobilizer patients (mean \pm SD $1.6 \pm 4.8$, range 0-19).

Clinically, 3 of 10 patients within the nonmobilizer subgroup had one or more relapses during the first year of therapy: two of them discontinued the treatment, only one testing positive for serum antinatalizumab antibodies, indicating that lack of response to treatment is not well-explained by neutralizing mechanisms. In statistically significant contrast, none of the 17 mobilizer patients experienced relapses during the treatment $(p=0.04)$. The mean change in EDSS scores at 6 months of treatment showed opposite trends in the mobilizer group (mean EDSS change $=-0.20$, range $=-1,+0.5)$ and in the nonmobilizer group (mean EDSS change $=+0.15$, range $=-0.5,+1)$, although the difference was not statistically significant $(p=0.08)$. These observations support the association between a HSPC mobilizer patient classification with a treatment responder profile and between nonmobilizer classification and lack of response to natalizumab, both neuroradiologically and clinically.

Circulating B-cell frequency increases in natalizumabtreated patients with different naive/memory profiles depending on the HSPC mobilization status. To detect a potential association of HSPC mobilization with effects on adaptive immune homeostasis, we next examined the size of circulating $\mathrm{T}$ - and B-cell pools and some relevant subsets during the first year of treatment and assessed their possible relationship with the natalizumab-induced circulating HSPC increase in the same cohort of 29 patients that was assessed for HSPC cell cycle status; untreated patients with MS and HD served as controls.

The proportion of CD19+ B cells was significantly higher in all the natalizumab-treated groups compared to HD (figure 4A). Among the B-cell subsets, the frequency of immunoglobulin $\mathrm{D}(\mathrm{IgD})+$ CD27+CD19+ early memory (class unswitched) B cells was found increased in the prospective patients after the first infusion compared to baseline $(p<$ $0.05)$ whereas no significant changes were detected in the proportions of $\mathrm{IgD}+\mathrm{CD} 27+\mathrm{CD} 19+$ naive and $\mathrm{IgD}+\mathrm{CD} 27+\mathrm{CD} 19+$ class-switched memory 
Figure 4 Circulating B-cell frequency increases in natalizumab-treated patients with different naive/memory profile according to hematopoietic stem and progenitor cells mobilization status

A

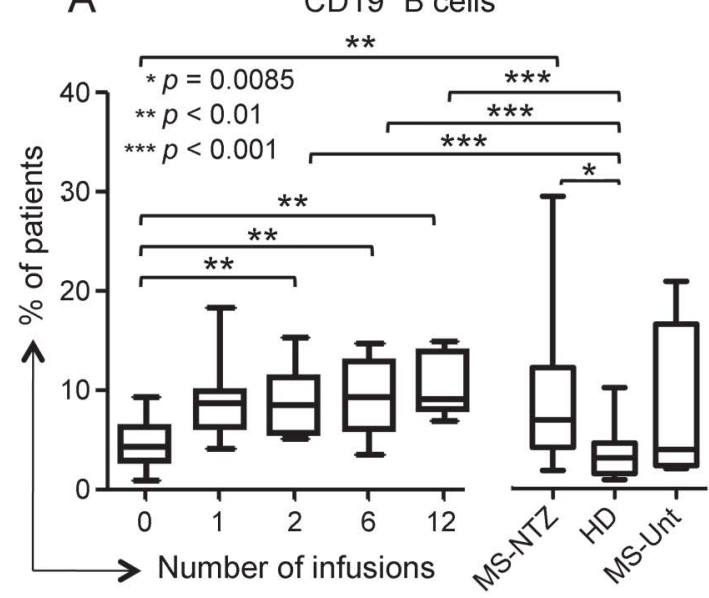

C $\quad \operatorname{lgD}{ }^{+} \mathrm{CD} 27^{+}$early memory $\mathrm{CD} 19^{+} \mathrm{B}$

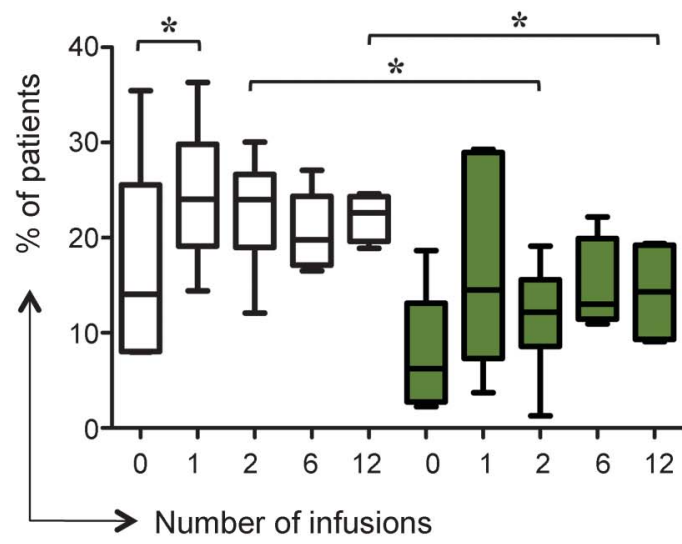

B

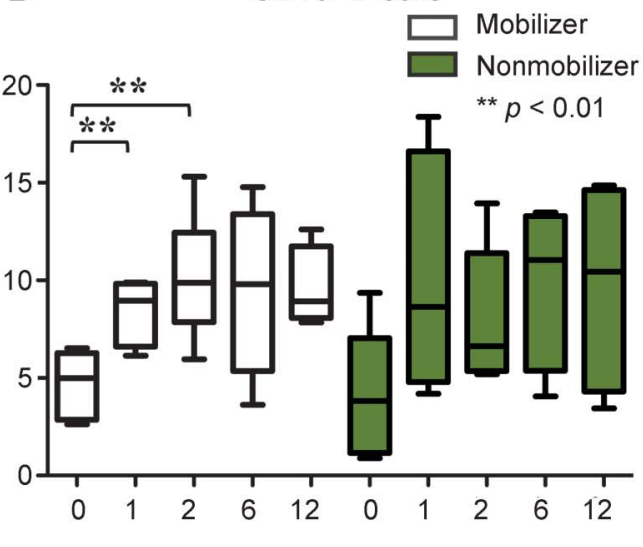

D IgD+CD27- naive $\mathrm{CD} 19^{+} \mathrm{B}$

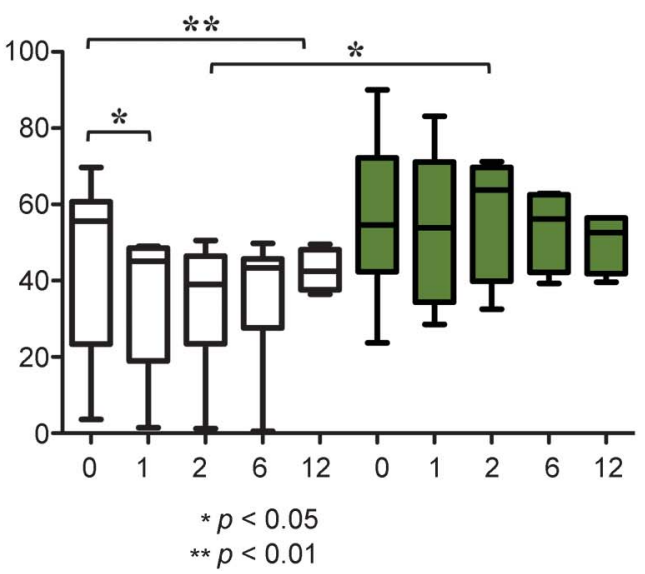

(A) CD19+ B cells proportion gradually increased during natalizumab after 1, 2, 6, and 12 infusions (mean percentages \pm SD $9.11 \pm 3.41 \%, 9.6 \pm 3.99 \%$, and $10.58 \pm 3.15 \%$, respectively) compared to baseline (mean \pm SD $4.81 \pm 2.67 \%$ ). Proportions are also significantly higher in the cross-sectional patients (multiple sclerosis [MS]-natalizumab [NTZ]; mean \pm SD $9.36 \pm 7.24 \%$ ) compared to the prospective ones at baseline and to the healthy donors (HD; mean \pm SD $3.79 \pm$ $2.79 \%)$. Untreated patients with MS (MS-Unt) are shown on the right. (B) CD19+ B cells proportion increased significantly only in the hematopoietic stem and progenitor cells (HSPC) mobilizer group of patients after 1 and 2 natalizumab infusions (mean \pm SD $8.41 \pm 1.58 \%$ and $10.71 \pm 3.2 \%$, respectively) compared to baseline (mean \pm SD $4.71 \pm 1.75 \%$ ). Mobilizer (open boxes) and nonmobilizer (shaded boxes) pattern legend applies also to $C$ and D. (C) Immunoglobulin D (IgD)+ CD27+CD19+ early memory B cells proportions were significantly higher in the mobilizer compared to the nonmobilizer patients after 2 (mean \pm SD: mobilizer 22.84\% \pm 6.04 ; nonmobilizer 11.7\% \pm 5.88 ) and 12 infusions (mean \pm SD: mobilizer $22.19 \pm 2.47 \%$; nonmobilizer $14.28 \pm 5.47 \%$ ). At pre- to post-treatment comparison within each subgroup, the proportion of IgD + CD27 + CD19+ early memory B cells was significantly increased only in the mobilizer patients after the first infusion (mean \pm SD $24.52 \pm 7.58 \%$ ) compared to baseline (mean \pm SD $16.97 \pm 10.69 \%$ ). (D) IgD+ CD27-CD19+ naive B cells percentages after 2 infusions were higher in the nonmobilizer patients (mean \pm SD 57.16 $\pm 15.9 \%$ ) than in the mobilizer ones (mean \pm SD $24.3 \pm 17.7 \%)$. At pre- to post-treatment comparison within each subgroup, the proportion of IgD + CD27-CD19+ naive B cells significantly decreased only in the mobilizer patients after 1 (mean \pm SD $35.65 \pm 19.04 \%$ ) and 12 infusions (mean \pm SD $42.72 \pm 5.45 \%$ ) compared to baseline (mean \pm SD 45.37 $\pm 24.25 \%)$, mirroring the change observed in the early memory B-cell proportions at the same time point.

B cells, either longitudinally in the natalizumabtreated patients or by comparing the natalizumabtreated groups with the untreated patients and HD (data not shown).

When stratifying the patient population according to HSPC mobilization response, we observed a significant increase of CD19+ B-cell proportion only in the mobilizer subgroup (6 of 12 patients) after 1 and 2 infusions compared to baseline $(p<0.05$; figure $4 \mathrm{~B}$ ). More interestingly, there was a consistent trend to a higher proportion of early memory $\mathrm{B}$ cells in the mobilizer than in the nonmobilizer subgroup at 
all time points, significant after 2 and 12 infusions (figure $4 \mathrm{C}$ ). Mirroring these changes, the proportion of naive $\mathrm{B}$ cells decreased in the mobilizer patients at all treatment time points compared to baseline, reaching significance after 1 and 12 infusions (respectively $p<0.05$ and $p<0.01$; figure 4D). No significant changes were seen in the nonmobilizer patients, and after 2 natalizumab infusions their mean naive B-cell proportion was twice the size of the mobilizer subgroup $(p<0.05$; figure $4 \mathrm{D})$.

The proportion of $\mathrm{CD} 103+\mathrm{CD} 8+$ regulatory $\mathrm{T}$ cells significantly increases in natalizumab-treated patients according to HSPC mobilization status.

The percentage of $\mathrm{CD} 103+\mathrm{CD} 8+\mathrm{T}$ cells, a described regulatory cell phenotype, ${ }^{15,16}$ gradually and significantly increased after $2(p<0.05), 6$ $(p<0.01)$, and $12(p<0.001)$ infusions compared to baseline in the 12 prospectively evaluated patients, and it was significantly higher at the 2 latter time points compared to $\mathrm{HD}$ (figure $5 \mathrm{~A}$ ). The frequency of this specific $\mathrm{CD} 8 \mathrm{~T}$-cell population increased more significantly than the total CD8 and CD4 T-cell subsets and remained elevated after 12 infusions, whereas CD8 and CD4 T-cell proportions showed a downward trend at that timepoint (figure e-1, A and B). The increase of $\mathrm{CD} 103+\mathrm{CD} 8+\mathrm{T}$ cells was especially pronounced in the mobilizer patients ( 6 of 12 patients; figure 5B). Although the total CD8 T-cell proportions in both patient strata did not change after treatment (figure e-1, C and D), the putative regulatory $\mathrm{CD} 103+\mathrm{CD} 8+\mathrm{T}$ cells appeared to accumulate in the mobilizer patients during treatment and their frequency was significantly higher than in the nonmobilizer patients after 12 natalizumab infusions $(p<0.01$; figure 5B).

DISCUSSION In this study, we sought to clarify some of the mechanisms underlying the HSPC increase observed in patients with MS during natalizumab treatment by investigating the HSPC cell cycle status and differentiation potential. We also examined the functional relevance in MS of the increase in circulating HSPC number by investigating its association with outcomes of disease activity and with changes in the homeostasis of immune cell subsets.

Our cell cycle analysis demonstrated not only quiescence (G0 state) of natalizumab-induced HSPC consistently with one previous report ${ }^{12}$ but also an increased proportion and significantly greater homogeneity of distribution of quiescent HSPC in the PB of natalizumab-treated patients compared to untreated patients and to HD. Thus this evidence strongly suggests an augmented rate of egress from the bone marrow, therefore a true mobilization effect of the drug, as hypothesized by us and previously, ${ }^{17}$ yet does not rule out the potential contribution of a natalizumab-mediated impaired homing of HSPC to the bone marrow. ${ }^{18}$ Moreover, we confirmed the healthy state of the bone marrow compartment in MS and showed that natalizumab-mobilized HSPC retain full capacity to expand and differentiate; therefore their increase in the circulation may have biological effects.

In one study, natalizumab-mobilized HSPC have been speculated to contribute to the pathogenesis of progressive multifocal encephalopathy (PML) by carrying JC virus, ${ }^{19}$ yet the authors report the presence of JCV DNA also in HD HSPC (17\%) and did not look at prospective HSPC counts, thus fall short of providing compelling evidence to support causation of PML by natalizumab-mobilized HSPC. Two other studies have demonstrated that natalizumabmobilized CD34+ HSPC are not a relevant reservoir for the virus. ${ }^{18,20}$ Technical reasons and different number of patients and of natalizumab infusions may account for the discrepancies among the studies, as discussed also in Chalkias et al., ${ }^{21}$ and it remains controversial whether CD34+ HSPC mobilization takes part in the development of PML.

Our prospective study not only confirms the previously reported natalizumab-induced HSPC increase ${ }^{12,13}$ but also adds new knowledge by demonstrating a widely different HSPC mobilization response among patients. The heterogeneity detected in the HSPC mobilization response enabled us to stratify patients as mobilizer or nonmobilizer and to examine clinical and immunologic correlations. Through this approach we demonstrate a significant association between nonmobilizer status and lack of response to natalizumab as well as differential effects on the size of the pools of circulating naive and memory $\mathrm{B}$ cells and of regulatory $\mathrm{T}$ cells, compared to the mobilizer condition.

The persistence and higher number of active brain lesions in the nonmobilizer subgroup at 6-month follow-up MRI compared to the mobilizer subgroup, as well as the exclusive occurrence of relapses and the predominant occurrence of worsening disability in the former subgroup, suggest that poor HSPC mobilization precedes and may predict failure to respond to natalizumab. The data from our small patients cohort seem to indicate a lower proportion of patients free from gadolinium-enhancing lesions $(62.96 \%)$ at 1 year as compared to the Natalizumab Safety and Efficacy in Relapsing-Remitting Multiple Sclerosis (AFFIRM) and Safety and Efficacy of Natalizumab in Combination With Interferon Beta-1a in Patients With Relapsing-Remitting Multiple Sclerosis (SENTINEL) studies (96\%). ${ }^{3,4,22}$ The proportion of relapse-free patients, however, was larger in our cohort (88.8\%) compared to the AFFIRM (77\%) and SENTINEL (72\%) studies. Our clinical results 
Figure 5 CD103+CD8+ regulatory $T$ cells proportion significantly increases in natalizumab-treated patients to a different extent according to hematopoietic stem and progenitor cells mobilization status

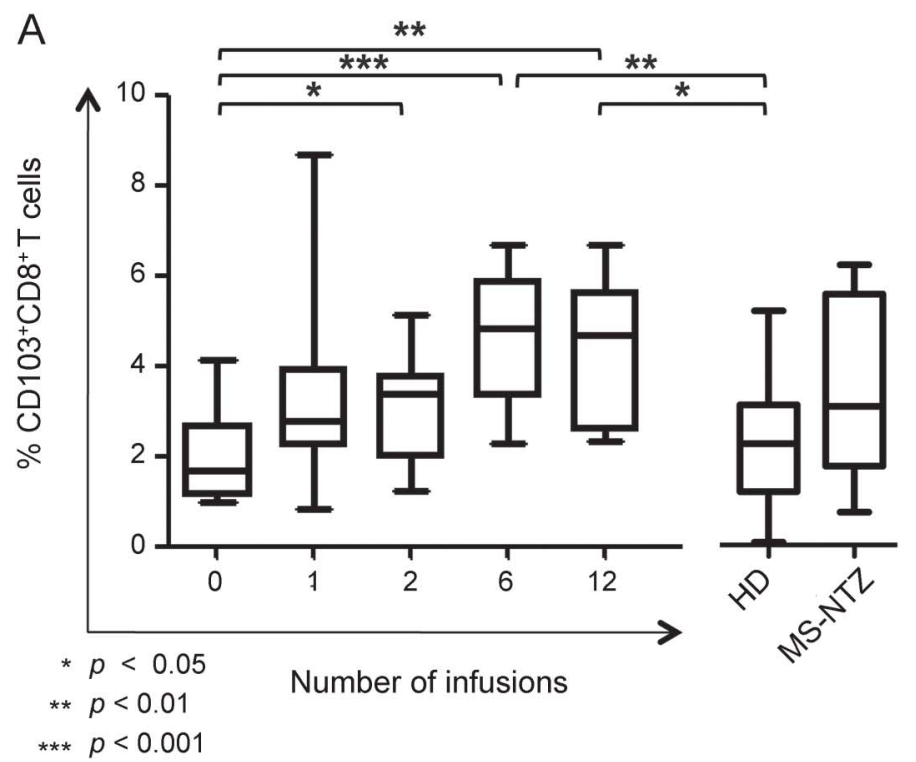

$\mathrm{B}$

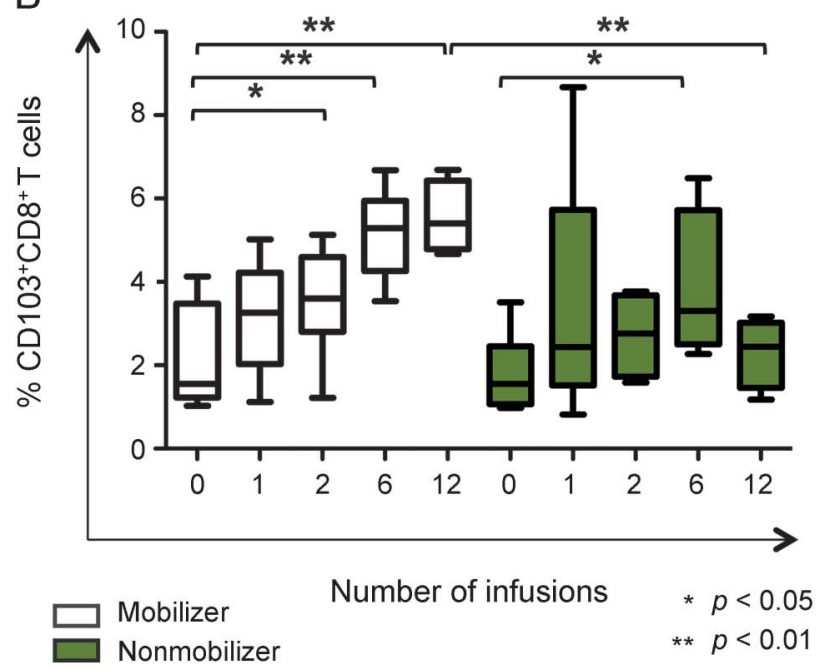

(A) The proportion of circulating CD103 + CD8 + T regulatory cells increased in patients with multiple sclerosis (MS) after 2, 6, and 12 natalizumab infusions (mean \pm SD $3.13 \pm 1.17 \%$, $4.63 \pm 1.48 \%$, and $4.31 \pm 1.66 \%$, respectively) compared to pretreatment baseline (mean \pm SD $1.94 \pm 1.03 \%$ ). In the patients who had received 6 and 12 infusions, CD103+CD8+ T cells proportion was significantly higher compared to healthy donors (HD; mean \pm SD 2.21 $\pm 1.28 \%$ ). The cross-sectional group of natalizumab-treated patients with MS (MS-NTZ) is represented on the right side. (B) $\mathrm{CD} 103+\mathrm{CD} 8+\mathrm{T}$ regulatory cells proportion gradually increased during the first year of natalizumab treatment and in mobilizer patients was significantly higher after 2, 6, and 12 infusions (mean \pm SD $3.55 \pm 1.32 \%, 5.15 \pm 1.06 \%$, and $5.54 \pm 0.86 \%$, respectively) compared to baseline (mean \pm SD $2.13 \pm 1.25 \%$ ). Nonmobilizer patients showed a significantly higher percentage of CD103+CD8+ T cells only after 6 infusions compared to pretreatment values and more importantly after 12 infusions they had significantly lower proportions of $\mathrm{CD} 103+\mathrm{CD} 8+\mathrm{T}$ cells (mean $\pm \mathrm{SD} 2.31 \pm 0.83 \%$ ) than mobilizer patients.

are consistent with postmarketing cohort studies showing that natalizumab is more effective in patients with milder disability (EDSS $\leq 2.5^{23}$ or between 3 and $3.5^{24}$ ) and very active disease ${ }^{24}$ and also in line with the results of subgroup analysis of the AFFIRM

and SENTINEL trials indicating a more significant efficacy of natalizumab in patients with higher disease activity ( $\geq 2$ relapses in the 12 months before starting the treatment) and who did not previously receive other disease-modifying therapies (DMT). ${ }^{25}$ The baseline clinical characteristics of our nonmobilizer subgroup, which includes the majority of the patients with persistent disease activity, showed a trend to lower number of relapses in the year before starting the treatment, higher EDSS score, longer disease duration, and higher number of previously received DMT (table e-2). These features suggest, even in the presence of underlying MRI activity, a more progressive MS profile of the nonmobilizer patients, which may also have a biological role in determining HSPC response but per se did not allow prediction of the response to treatment. Early HSPC counts, therefore, may be useful as a predictive biomarker of risk of persistent MRI and clinical disease activity during the first year of treatment with natalizumab. Further studies in a larger prospective cohort are required to confirm these results and define the optimal cutoff for stratification of patients according to HSPC mobilization at an early stage during treatment with natalizumab in order to identify those at higher risk of persistence of disease activity.

Our study provides further insight into the increase of circulating $B$ cells previously shown during natalizumab ${ }^{26,27}$ by demonstrating a specific profile of naive/memory phenotype redistribution depending on the HSPC mobilization response. We report a more significant increase of total $\mathrm{B}$ cells among the HSPC mobilizer patients and show predominance of early memory B cells in this patient subgroup and of naive $B$ cells in the nonmobilizer subgroup, suggesting that in the latter individuals memory B cells might still be able to extravasate and cross the blood-brain barrier to access the CNS, potentially contributing to persistence of disease activity. This interpretation is consistent with the natalizumab-induced increase in marginal zone-like (early memory) B cells recently reported $^{28}$ and is in line with the increased proportions of B-memory subsets previously described in natalizumab-treated patients. ${ }^{29}$

We observed a small increase in the proportion of circulating mature $\mathrm{T}$ lymphocytes during natalizumab, similarly to previously published data, ${ }^{10}$ and potentially associated with protection from clinical reactivation postnatalizumab discontinuation. ${ }^{30}$ In notable addition, we report a significant and persistent accumulation of the proportion of $\mathrm{CD} 103+\mathrm{CD} 8+\mathrm{T}$ cells, described as a regulatory population ${ }^{15,16}$ and more pronounced among the mobilizer patients, thus suggesting a novel immune tolerizing mechanism, previously described after HSPC transplantation in patients with systemic lupus erythematosus ${ }^{31}$ but not in 
natalizumab-treated MS. Therefore, the status of HSPC mobilizers is associated with posttreatment selective changes in lymphocyte subpopulations that likely have functional significance.

Our data suggest that increased circulating HSPC numbers reflect active export from the bone marrow and may be of value in predicting the clinical response to natalizumab. We propose that, if validated in additional studies, the HSPC number fold increase after 2 natalizumab infusions could be clinically useful as an early biomarker of efficacy of natalizumab treatment. We envision that this predictor of efficacy could usefully complement other patient stratification strategies, currently mainly focused on estimating the risk of PML during natalizumab therapy and on the risk of MS reactivation after discontinuation, and would therefore represent an advance towards multidimensional personalized treatment decisions.

\section{AUTHOR CONTRIBUTIONS}

Miriam Mattoscio: conception and design, collection and assembly of data, data analysis and interpretation, manuscript writing, critical revision of the manuscript for important intellectual content, final approval of manuscript. Richard Nicholas: provision of patients, data interpretation, manuscript writing, critical revision of the manuscript for important intellectual content, final approval of manuscript. Maria Pia Sormani: data analysis and interpretation, critical revision of the manuscript for important intellectual content, final approval of manuscript. Omar Malik: provision of patients, critical revision of the manuscript for important intellectual content, final approval of manuscript. Jean Lee: data analysis, critical revision of the manuscript for important intellectual content, final approval of manuscript. Adam Waldman: data analysis, critical revision of the manuscript for important intellectual content, final approval of manuscript. Francesco Dazzi: data analysis and interpretation, critical revision of the manuscript for important intellectual content, final approval of manuscript. Paolo A. Muraro: conception and design, provision of study material and patients, collection and assembly of data, data analysis and interpretation, manuscript writing, critical revision of the manuscript for important intellectual content, final approval of manuscript.

\section{ACKNOWLEDGMENT}

The authors thank Dr. Sofia Abrahamsson from Imperial College London and Dr. Benedetta Mazzanti and Dr. Riccardo Saccardi from Careggi Hospital, Florence, Italy, for their scientific and technical advice; Caroline D'Arcy, Sinead O'Driscoll, Johnny De la Cruz, Ferdinand Pacuribot, Theresa Felongco, and Lolena Parreno, MS Specialist nurses at Charing Cross Hospital, London, UK, for their assistance with the study; the patients with MS and healthy subjects who donated their blood for this study; and the NIHR/Wellcome Trust Imperial CRF and Imperial College London Biomedical Research Centre for their logistic support.

\section{STUDY FUNDING}

Supported by the Italian MS Society (FISM; project grant ref. no. 2010/ R/24 to P.A. Muraro, training fellowships ref. no. 2010/B/10 and 2012/ B/8 to M. Mattoscio) and the Medical Research Council (ref. no. G0800679 to P.A.M.). Pilot funding was provided by the Multiple Sclerosis Trial Collaboration (a registered UK Charity). This article presents independent research funded by the Italian MS Society and the MRC and partially carried out at the NIHR/Wellcome Trust Imperial CRF.

\section{DISCLOSURE}

M. Mattoscio has been supported via industry-funded travel by Biogen Idec, Teva, and Genzyme. R. Nicholas has received honoraria for speaking engagements and for serving on editorial advisory boards from Merck-Serono, Novartis, and Biogen Idec. He has received funding from
Biogen Idec. He has received support for travel to conferences from Novartis and Biogen Idec. M. Sormani reports personal fees from MerckSerono, Biogen Idec, Teva, Actelion, Synthon, and Novartis, all unrelated to the submitted work. O. Malik has received honoraria and travel bursaries from Biogen Idec and Novartis. J. Lee, A. Waldman, F. Dazzi, and P. Muraro report no disclosures relevant to the manuscript. Go to Neurology.org for full disclosures.

Received October 2, 2014. Accepted in final form December 22, 2014.

\section{REFERENCES}

1. Rose DM, Alon R, Ginsberg MH. Integrin modulation and signaling in leukocyte adhesion and migration. Immunol Rev 2007;218:126-134.

2. Miller DH, Soon D, Fernando KT, et al. MRI outcomes in a placebo-controlled trial of natalizumab in relapsing MS. Neurology 2007;68:1390-1401.

3. Polman CH, O'Connor PW, Havrdova E, et al. A randomized, placebo-controlled trial of natalizumab for relapsing multiple sclerosis. N Engl J Med 2006;354: 899-910.

4. Radue EW, Stuart WH, Calabresi PA, et al. Natalizumab plus interferon beta-1a reduces lesion formation in relapsing multiple sclerosis. J Neurol Sci 2010;292:28-35.

5. Phillips JT, Giovannoni G, Lublin FD, et al. Sustained improvement in expanded disability status scale as a new efficacy measure of neurological change in multiple sclerosis: treatment effects with natalizumab in patients with relapsing multiple sclerosis. Mult Scler 2011;17:970-979.

6. Zivadinov R, Dwyer MG, Hussein S, et al. Voxel-wise magnetization transfer imaging study of effects of natalizumab and IFNbeta-1a in multiple sclerosis. Mult Scler 2012;18:1125-1134.

7. Krysko KM, O'Connor PW. The Toronto observational study of natalizumab in multiple sclerosis. Can J Neurol Sci 2011;38:422-428.

8. Stuve O, Marra CM, Bar-Or A, et al. Altered $\mathrm{CD} 4+/ \mathrm{CD} 8+\mathrm{T}$-cell ratios in cerebrospinal fluid of natalizumab-treated patients with multiple sclerosis. Arch Neurol 2006;63:1383-1387.

9. Stuve O, Marra CM, Jerome KR, et al. Immune surveillance in multiple sclerosis patients treated with natalizumab. Ann Neurol 2006;59:743-747.

10. Kivisakk P, Healy BC, Viglietta V, et al. Natalizumab treatment is associated with peripheral sequestration of proinflammatory T cells. Neurology 2009;72:1922-1930.

11. Stuve O. The effects of natalizumab on the innate and adaptive immune system in the central nervous system. J Neurol Sci 2008;274:39-41.

12. Bonig $\mathrm{H}$, Wundes A, Chang $\mathrm{KH}$, Lucas $\mathrm{S}$, Papayannopoulou T. Increased numbers of circulating hematopoietic stem/progenitor cells are chronically maintained in patients treated with the CD49d blocking antibody natalizumab. Blood 2008;111:3439-3441.

13. Zohren F, Toutzaris D, Klarner V, Hartung HP, Kieseier B, Haas R. The monoclonal anti-VLA-4 antibody natalizumab mobilizes CD34+ hematopoietic progenitor cells in humans. Blood 2008;111:3893-3895.

14. Mendez-Ferrer S, Lucas D, Battista M, Frenette PS. Haematopoietic stem cell release is regulated by circadian oscillations. Nature 2008;452:442-447.

15. Ho J, Kurtz CC, Naganuma M, Ernst PB, Cominelli F, Rivera-Nieves J. A CD8+/CD103high T cell subset regulates TNF-mediated chronic murine ileitis. J Immunol 2008;180:2573-2580. 
16. Uss E, Rowshani AT, Hooibrink B, Lardy NM, van Lier RA, ten Berge IJ. CD103 is a marker for alloantigeninduced regulatory CD8+ T cells. J Immunol 2006;177: 2775-2783.

17. Jing D, Oelschlaegel U, Ordemann R, et al. CD49d blockade by natalizumab in patients with multiple sclerosis affects steady-state hematopoiesis and mobilizes progenitors with a distinct phenotype and function. Bone Marrow Transplant 2010;45:1489-1496.

18. Saure C, Warnke C, Zohren F, et al. Natalizumab and impedance of the homing of CD34+ hematopoietic progenitors. Arch Neurol 2011;68:1428-1431.

19. Frohman EM, Monaco MC, Remington G, et al. JC virus in CD34+ and CD19+ cells in patients with multiple sclerosis treated with natalizumab. JAMA Neurol 2014;71:596-602.

20. Warnke C, Smolianov V, Dehmel T, et al. CD34+ progenitor cells mobilized by natalizumab are not a relevant reservoir for JC virus. Mult Scler 2010;17:151-156.

21. Chalkias S, Dang X, Bord E, et al. JC virus reactivation during prolonged natalizumab monotherapy for multiple sclerosis. Ann Neurol 2014;75:925-934.

22. Rudick RA, Stuart WH, Calabresi PA, et al. Natalizumab plus interferon beta-1a for relapsing multiple sclerosis. $\mathrm{N}$ Engl J Med 2006;354:911-923.

23. Prosperini L, Gianni C, Barletta V, et al. Predictors of freedom from disease activity in natalizumab treated-patients with multiple sclerosis. J Neurol Sci 2012;323:104-112.

24. Laroni A, Gandoglia I, Solaro C, et al. Clinical baseline factors predict response to natalizumab: their usefulness in patient selection. BMC Neurol 2014;14:103.
25. Hutchinson M, Kappos L, Calabresi PA, et al. The efficacy of natalizumab in patients with relapsing multiple sclerosis: subgroup analyses of AFFIRM and SENTINEL. J Neurol 2009;256:405-415.

26. Krumbholz M, Meinl I, Kumpfel T, Hohlfeld R, Meinl E. Natalizumab disproportionately increases circulating pre-B and B cells in multiple sclerosis. Neurology 2008;71: 1350-1354.

27. Lesesve JF, Debouverie M, Decarvalho Bittencourt M, Bene MC. CD49d blockade by natalizumab therapy in patients with multiple sclerosis increases immature B-lymphocytes. Bone Marrow Transplant 2011;46: 1489-1491.

28. Planas R, Jelcic I, Schippling S, Martin R, Sospedra M. Natalizumab treatment perturbs memory- and marginal zone-like B-cell homing in secondary lymphoid organs in multiple sclerosis. Eur J Immunol 2012;42:790-798.

29. Haas J, Bekeredjian-Ding I, Milkova $M$, et al. B cells undergo unique compartmentalized redistribution in multiple sclerosis. J Autoimmun 2011;37:289-299.

30. Rossi S, Motta C, Studer V, et al. A genetic variant of the anti-apoptotic protein Akt predicts natalizumab-induced lymphocytosis and post-natalizumab multiple sclerosis reactivation. Mult Scler 2013;19:59-68.

31. Zhang L, Bertucci AM, Ramsey-Goldman R, Burt RK, Datta SK. Regulatory T cell (Treg) subsets return in patients with refractory lupus following stem cell transplantation, and TGF-beta-producing CD8 + Treg cells are associated with immunological remission of lupus. J Immunol 2009;183:6346-6358.

\section{This Week's Neurology ${ }^{\circledR}$ Podcast}

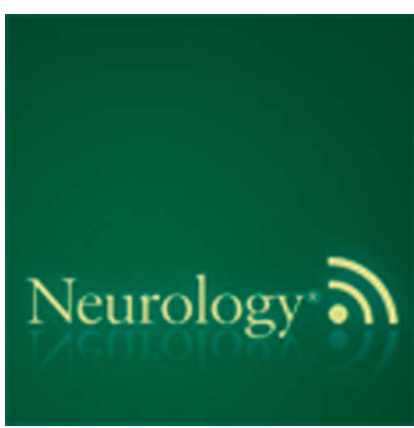

Quality improvement in neurology: Epilepsy Update Quality Measurement Set

This podcast begins and closes with Dr. Robert Gross, Editor-inChief, briefly discussing highlighted articles from the April 7, 2015, issue of Neurology. In the second segment, Dr. Joanna Suski talks with Dr. Nathan Fountain about his paper on the update of quality measures in epilepsy. Dr. Sarah Wesley then reads the e-Pearl of the week about hemicrania continua. In the next part of the podcast, Dr. Ted Burns focuses his interview with Drs. Radhika Dhamija and Christopher Klein on the topic of next generation sequencing.

Disclosures can be found at Neurology.org.

At Neurology.org, click on "RSS" in the Neurology Podcast box to listen to the most recent podcast and subscribe to the RSS feed.

CME Opportunity: Listen to this week's Neurology Podcast and earn 0.5 AMA PRA Category 1 CME Credits ${ }^{\mathrm{TM}}$ by answering the multiple-choice questions in the online Podcast quiz. 